\title{
Lipemic Serum in Hypertriglyceridemia-Induced Pancreatitis
}

\author{
Michael A. Santos, $M D^{1,2,3}$, Nisarg B. Patel, $M D^{2}$, and Carolina Correa, $M D^{2}$ \\ 'Department of Medicine, Penn State Milton S Hershey Medical Center, Hershey, PA, USA; ${ }^{2}$ WellSpan Good Samaritan Hospital, Lebanon, PA, USA; \\ ${ }^{3}$ Department of Medicine, WellSpan Good Samaritan Hospital, Lebanon, PA, USA.
}

KEY WORDS: clinical image; hypertriglyceridemia; acute pancreatitis; lipemic.

J Gen Intern Med 32(11): 1267

DOI: $10.1007 / \mathrm{s} 11606-017-4086-y$

(c) Society of General Internal Medicine 2017

A 47-year-old male with hypertension presented with epigastric pain. He did not have gallstones and rarely drank alcohol. Physical examination revealed tachycardia and epigastric tenderness. Abdominal CT scan showed pancreatic stranding. Serum triglycerides were $3568 \mathrm{mg} / \mathrm{dl}$ (range 0-

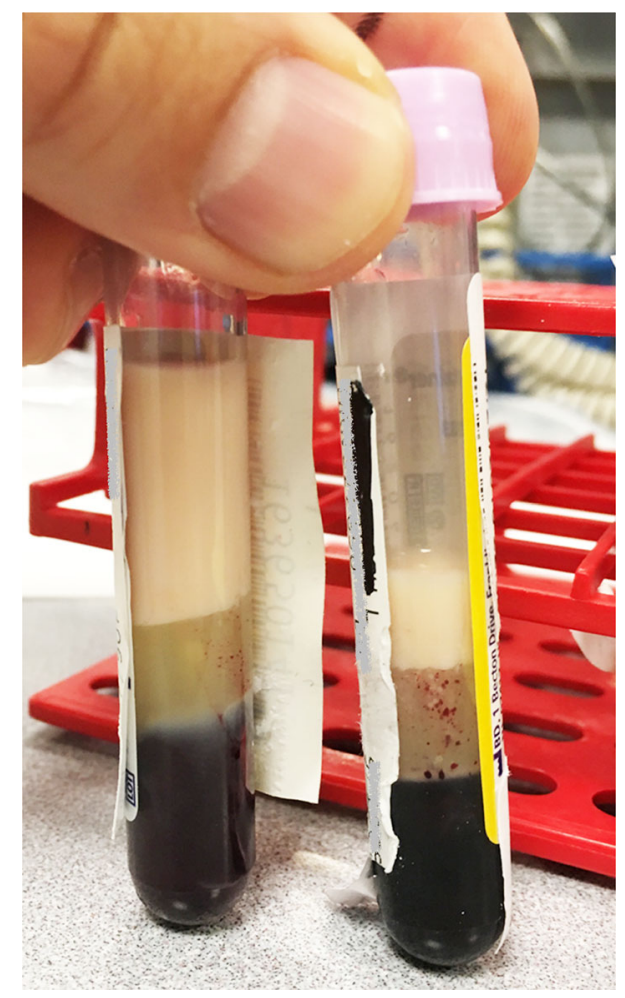

Figure 1 Unused blood samples showing a gross reduction in lipid volume from admission and after $12 \mathrm{~h}$ of IV insulin. After centrifugation, the lipemic layers settle above the gel separator layer

Received March 2, 2017

Revised April 12, 2017

Accepted May 17, 2017

Published online June 2, 2017
$150 \mathrm{mg} / \mathrm{dl})$. He was diagnosed with hypertriglyceridemiainduced pancreatitis (HTGP). After $12 \mathrm{~h}$ of intravenous insulin at $0.1 \mathrm{units} / \mathrm{kg} / \mathrm{h}$, a reduction in triglycerides was visually apparent (Fig. 1).

Severe hypertriglyceridemia is the third most common cause of pancreatitis, after gallstones and alcohol. ${ }^{1}$ Acute pancreatitis is rare when serum triglycerides are less than $1000 \mathrm{mg} / \mathrm{dl}$. The pathophysiology of HTGP is unclear. Proposed mechanisms include increased blood viscosity from elevated levels of chylomicrons and the aggregation of free fatty acids, with subsequent pancreatic capillary occlusion leading to ischemic acinar cell injury. ${ }^{2}$

Acute management is supportive, with intravenous insulin, fluids, and dextrose until serum triglycerides are below $500 \mathrm{mg} / \mathrm{dl}$. Insulin increases lipoprotein lipase activity, promoting chylomicron breakdown and clearance of serum triglycerides. ${ }^{3}$ Heparin with insulin can be considered, but heparin monotherapy is not recommended. ${ }^{4}$ When feasible, early plasmapheresis can reduce serum triglycerides by $65-70 \%$ in a single session. ${ }^{5}$ Clinicians should also focus on dietary modification with early initiation of fibrates, niacin, and fish oil.

Corresponding Author: Michael A. Santos, MD; Department of MedicineWellSpan Good Samaritan Hospital, Lebanon, PA, USA (e-mail: masantospitt@gmail.com).

Compliance with ethical standards:

Funding: None.

Prior Presentations: None.

Conflict of Interest: The authors declare that they do not have a conflict of interest.

\section{REFERENCES}

1. Toskes PP. Hyperlipidemic pancreatitis. Gastroenterol Clin North Am. 1990; 19(4):783-791.

2. Valdivielso P, Ramirez-Bueno A, Ewald N. Current knowledge of hypertriglyceridemic pancreatitis. Eur J Intern Med. 2014;25(8):689-694.

3. Goldberg IJ. Lipoprotein lipase and lipolysis: central roles in lipoprotein metabolism and atherogenesis. J Lipid Res. 1996;37(4):693-707.

4. Berglund L, Brunzell JD, Goldberg AC, et al. Evaluation and treatment of hypertriglyceridemia: an Endocrine Society clinical practice guideline. J Clin Endocrinol Metab. 2012;97(9):2969-2989.

5. Lennertz A, Parhofer KG, Samtleben W, Bosch T. Therapeutic plasma exchange in patients with chylomicronemia syndrome complicated by acute pancreatitis. Ther Apher. 1999;3(3):227-233. 